\title{
Physicochemical Properties, Chemical Compositions and Antioxidant Activities of Rhizome Oils from Two Varieties of Kaempferia galanga
}

\author{
I Wayan Muderawan*, I Wayan Mudianta, and Ni Wayan Martiningsih \\ Department of Chemistry, Faculty of Mathematics and Natural Sciences, Ganesha University of Education, \\ Singaraja 81117, Bali, Indonesia
}

* Corresponding author:

email:wayan.muderawan@undiksha.ac.id

Received: June 4, 2021

Accepted: October 12, 2021

DOI: $10.22146 / \mathrm{ijc} .66348$

\begin{abstract}
Kaempferia galanga is a tropical plant with an impressive range of food and medicinal uses. This study, therefore, investigated the variation in yields, physicochemical properties, chemical compositions, and antioxidant activities of rhizome oils from two K. galanga varieties, K. galanga bigger rhizomes (V1) and $\mathrm{K}$. galanga smaller rhizomes (V2), isolated by steam distillation (S) and maceration (M) techniques. The air-dried rhizomes' oil contents were found to be $2.81 \pm 0.09 \%$

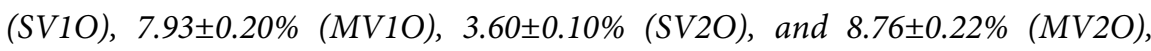
respectively. From the GC-MS analysis, the SV1O, MV1O, SV2O, and MV2O samples contain 49, 48, 61, and 56 compounds, respectively. Furthermore, ethyl trans-pmethoxycinnamate was the most prevalent chemical constituent in four oils with a percentage contribution of $43.37 \%$ (SV1O), 60.62\% (MV1O), 24.92\% (SV2O), and $57.17 \%$ (MV2O). Several long-chain alcohols (6Z,9Z-pentadeca-6,9-dien-1-ol, 9E,12E-octadeca-9,12-dien-1-ol, heptadecan-1-ol), aldehyde (Z-octadec-9-enal), carboxylic acids (4-(4-methoxyphenyl)oxane-4-carboxylic acid, hexadecanoic acid), diterpene sandaracopimaradiene, steroid ergosterol, and alkaloid 2-imino-3-(3nitrophenyl)-1,3-thiazolidin-4-one, were also identified in $\mathrm{K}$. galanga rhizome oils isolated by maceration method. In addition, all oils showed high antioxidant activities with the $I C_{50}$ values of $86.10 \pm 1.51,85.24 \pm 1.48,89.19 \pm 1.72$, and $86.49 \pm 2.03$ $\mu \mathrm{g} / \mathrm{mL}$ for SV1O, MV1O, SV2O, and MV2O, respectively.
\end{abstract}

Keywords: Kaempferia galanga; rhizome oil; physicochemical properties; chemical compositions; antioxidant activities

\section{- INTRODUCTION}

Kaempferia galanga Linn, known in Indonesia as 'kencur', is an aromatic plant belonging to the Zingiberaceae family, with broadly ovate and pale green leaves. This aromatic ginger leaves and rhizomes are commonly used as food flavoring agents and perfumery and prescribed as a traditional treatment against asthma, hypertension, malaria, and bronchitis [1-2]. In Traditional Chinese Medicines (TCM), the plant treats cholera, contusion, constipation, and stomachache. Meanwhile, the Indian Ayurvedic formulation suggested the plant's use for muscular swelling and rheumatism treatments [3]. Furthermore, the leaves have higher phenolic content, antioxidant activity, and metal ion- chelating ability than $K$. galanga rhizomes [4]. The rhizomes are also used to prepare 'Jamu beras kencur' in Indonesia, as a local tonic frequently consumed for beneficial health effects [5].

Srivastava et al. [6] recently reported on the physicochemical values and nutritional composition of K. galanga rhizomes. However, the rhizome oil's detailed chemical composition is scarce, especially oils obtained from the Indonesian cultivars. Also, reports on the plant's chemical and bioactivities are dominated by specimens collected from India [6], Bangladesh [7], Thailand [8-9], and Malaysia [10]. Most studies also investigated the rhizomes' polar extracts, using methanol and ethanol as major solvents. For instance, the ethanolic extract of $K$. galanga from Thailand

I Wayan Muderawan et al. 
displayed moderate cytotoxic activity against human tumor cell lines [8]. In contrast, the methanolic rhizome extract of the plant collected in India was effective against acute inflammation in rats.

Interestingly, ethyl-p-methoxycinnamate was discovered to be the extracts' major phytoconstituents [11]. Thus, only a few studies have investigated the rhizome extracts' non-polar fraction, including the report by Othman et al. [10], on organic extract preparation, using Soxhlet extractor, with petroleum ether and dichloromethane. Recently, Srivastava et al. [6] isolated a K. galanga rhizome oil using the hydrodistillation method, and ethyl trans-p-methoxycinnamate was purified as the major volatile component.

Currently, there are 10 different $K$. galanga cultivars domesticated in Indonesia. However, these are simply grouped into two types: the bigger rhizome, usually with wide leaves, dark brown skin, and the smaller counterpart, with narrow leaves and light brown skin [12]. The bigger rhizomes are widely cultivated in West Java, while the smaller counterparts are mostly grown in Central and East Java regions. Also, larger rhizomes have higher productivity (12-16 tons/ha) compared to small counterparts (6-8 tons/ha) but lower essential oil content [13].

Methanolic extracts of $K$. galanga rhizomes have been reported to have antioxidant activities [4,7]. Antioxidants are secondary metabolites produced by plants to protect against oxidative damage. Thus, they are completely essential for maintaining optimal cellular and systemic health and well-being. In the family Zingiberaceae, it is generally believed that antioxidants produced by the plant are transported to the rhizomes, where they are accumulated [4]. This study, therefore, reports the physicochemical properties, chemical compositions, and antioxidant activities of bigger and smaller rhizomes varieties of $K$. galanga rhizome essential oils.

\section{- EXPERIMENTAL SECTION}

\section{Materials}

For this experiment, two Kaempferia galanga, L. varieties, bigger (a) and smaller (b) rhizomes, were collected from Bali. Fig. 1(a) shows variety-1 (V1) has broad leaves, bigger rhizomes with a dark-brown epidermis,
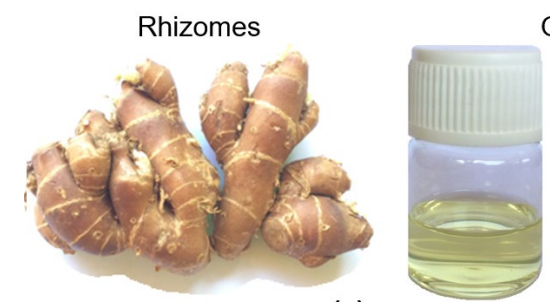

Oil

(a)

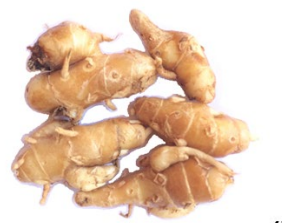

(b)

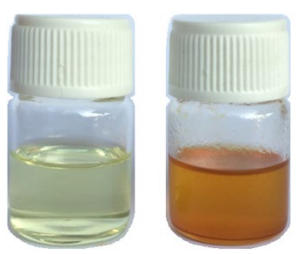

Fig 1. Two varieties of $K$. galanga, L. (a) bigger rhizomes with dark brown epidermis and the oils (SV1O and MV1O), and (b) smaller rhizomes with light brown epidermis and the oils (SV2O and MV2O)

$5.5-10.3 \mathrm{~cm}$ in length and $1.73-2.24 \mathrm{~cm}$ in diameter. Fig. 1(b) shows variety-2 (V2) has narrow leaves, a smaller rhizome with a light-brown epidermis, $4.0-7.2 \mathrm{~cm}$ length, and $1.5-1.7 \mathrm{~cm}$ diameter. The plant materials were then identified, and voucher specimens (KGR.01 and KGR.02) were deposited at the Herbarium of Biology Laboratory, Faculty of Mathematics and Natural Sciences, Ganesha University of Education, Singaraja Bali. Subsequently, the rhizomes were washed with water, cut into small pieces, air-dried at room temperature for 7 days, and ground to powder form. The powders were then stored in an air-tight container protected from light until further use.

\section{Procedure}

\section{Isolation of the rhizome oil}

Steam distillation. In this study, the steam distillation of essential oil from the K. galanga rhizomes employed a modified reported procedure [14]. First, the air-dried $K$. galanga rhizome powder (50 g) was steam distilled in Clevenger apparatus for $4 \mathrm{~h}$. Subsequently, the distillate was extracted with $n$-hexane $(3 \times 50 \mathrm{~mL})$, and the organic layer was dried with anhydrous sodium sulfate, left to stand overnight, filtered, as well as evaporated with a rotary vacuum evaporator. The essential oil obtained was then weighed to determine the mass, labeled SV1O and SV2O for the varieties 1 and 2, respectively, and stored to preserve freshness until use. 
Maceration. A modified reported procedure was used to maceration of oil from the K. galanga rhizomes [14]. For this process, air-dried $K$. galanga rhizome powder (50 g) was immersed in $n$-hexane $(250 \mathrm{~mL})$ for 3 days, and the mixture was separated by filtration. Subsequently, the residue was washed with $n$-hexane $(100 \mathrm{~mL})$ and combined with the filtrate. The light-yellow filtrate was then dried with sodium sulfate anhydrous, left overnight, filtered again, and evaporated using a rotary vacuum evaporator. It was followed by weighing the rhizome essential oil obtained, labeled as MV1O and MV2O for the varieties 1 and 2, respectively, and storage to preserve freshness until use.

\section{Determination of physicochemical properties}

Physicochemical characteristics provide a baseline for the suitability of oils [6,14-15]. The oil's physicochemical properties determined were percentage yield based on oil weight per weight of material used, color based on physical observation in daylight and under UV radiation using UV chamber, odor on organoleptic evaluation, solubility in water, hexane. Other properties include solubility, specific gravity on the pycnometer, refractive index on a refractometer, and optical activity on a polarimeter were also examined [14]. Meanwhile, the oils' acid value (AV), saponification value (SV), ester value $(\mathrm{EV})$, and iodine value (IV) were determined according to standard methods [15].

\section{Gas chromatography-mass spectrometry analysis}

Essential oil analysis was performed by gas chromatography coupled with mass spectrometry (GC/MS) in a GCMS-QP2010 Ultra, Shimadzu, equipped with an autosampler, AOC-20s, Shimadzu, auto-injector, AOC-20i, Shimadzu, and RTX-5MS (30 $\mathrm{m} \times 0.25 \mathrm{~mm}$ ID and $0.25 \mu \mathrm{m}$ film thickness) columns. The injector temperature was set at $200^{\circ} \mathrm{C}$. The oven temperature was initially at $70^{\circ} \mathrm{C}$ for $2 \mathrm{~min}$, programmed to reach $180^{\circ} \mathrm{C}$ at the rate of $20^{\circ} \mathrm{C} / \mathrm{min}$ and held at $180^{\circ} \mathrm{C}$ for $3 \mathrm{~min}$, then increased to $250{ }^{\circ} \mathrm{C}$ at the rate of $20^{\circ} \mathrm{C} / \mathrm{min}$, and finally kept constant at $250{ }^{\circ} \mathrm{C}$ for $16 \mathrm{~min}$. Helium was used as the carrier gas with a $35.2 \mathrm{~mL} / \mathrm{min}$ flow and $100 \mathrm{kPa}$ pressure. The sample $(0.2 \mu \mathrm{L})$ was neatly injected with a 20:1 split ratio, and the mass spectrometer was operated in the electron impact (EI) mode at $70 \mathrm{eV}$. Thus, the mass scanning range was varied over $35-500 \mathrm{~m} / \mathrm{z}$. Also, the ion source and quadrupole temperatures were set at 230 and $150^{\circ} \mathrm{C}$, respectively. The essential oil components were identified based on their mass spectral fragmentation using the Wiley $9 \mathrm{GC} / \mathrm{MS}$ libraries. Subsequently, the identified compound's percentage was computed from a total ion chromatogram [16].

\section{Antioxidant activity}

The free radical scavenging activity of the rhizome oil of K. galanga was determined spectrophotometrically. First, the rhizome oils' hydrogen atom or electron donation abilities were measured from the bleaching of purple-colored methanol solution of stable 2,2diphenyl-1-picrylhydrazyl radical (DPPH•) according to the reported procedure [17-18]. For this process, $0.5 \mathrm{~mL}$ of rhizome oil solution in $99.9 \%$ methanol, in the final concentration range of $0.025-0.250 \mathrm{mg} / \mathrm{mL}$, or $0.5 \mathrm{~mL}$ of methanol (control), were mixed with $3.5 \mathrm{~mL}$ of $100 \mu \mathrm{M}$ DPPH solution $(0.0039 \mathrm{~g}$ in $100 \mathrm{~mL}$ 99.9\% methanol prepared daily). Then, the mixture was vortexed thoroughly for a minute and left at room temperature for $30 \mathrm{~min}$, and the absorbance was read against control at $517 \mathrm{~nm}$, using a UV-2600 spectrophotometer (Shimadzu). The control probe contained all components except for the radicals. Subsequently, the oils' abilities to scavenge DPPH radicals, (DPPH• scavenging activity $\left(\mathrm{SA}_{\mathrm{DPPH}}.\right)$ ), was calculated using the equation:

$\mathrm{SA}_{\mathrm{DPPH}} \bullet(\%)=100 \times\left(\mathrm{A}_{\text {Control }}-\mathrm{A}_{\text {Sample }}\right) / \mathrm{A}_{\text {Control }}$

where $A_{\text {Control }}$ is the control reaction's absorbance (containing all reagents except the rhizome oil), and $\mathrm{A}_{\text {sample }}$ is the absorbance in the rhizome oil's presence. The oils' radical-scavenging ability was calculated as $\mathrm{IC}_{50}$ $(\mathrm{g} / \mathrm{mL})$ from the graph of the percentage of radical scavenging activity against the oil concentration.

\section{Statistical analysis}

All measurements were carried out in triplicate, and results were reported as mean $\pm \mathrm{SD}$, except for GCMS analysis. Furthermore, statistical analysis was performed using analysis of variance (ANOVA), and significant difference between sample averages $(p<0.05)$ 
was calculated using IBM SPSS Statistics software package version 23 .

\section{- RESULTS AND DISCUSSION}

\section{Physicochemical Properties of the Oils}

Studies of various physicochemical characteristics identify the practical importance and provide bases for the suitability and utility of various oils of plants origin in daily life. The physicochemical properties of the oil, including color, odor, solubility in water and organic solvents, specific gravity, refractive index, optical rotation, acid value, iodine value, and saponification value, indirectly affect the essential oil's quality.

In this study, oils obtained from two K. galanga varieties' rhizomes, isolated by steam distillation and maceration extraction, were evaluated for their physicochemical characteristics (Table 1). The oils isolated by steam distillation (SOs) were light yellow, transparent in physical state, and pleasant aromatic odor. At the same time, the maceration (MOs) counterparts were yellow, viscous, and had a pleasant aromatic odor. However, the oils had no difference in color and were both soluble in organic solvents, including $n$-hexane, ethanol, and diethyl ether, but insoluble in water.

The percent yields of oils obtained in this study were $2.81 \pm 0.09,7.93 \pm 0.20,3.60 \pm 0.10$, and $8.76 \pm 0.22$ for SV1O,
MV1O, SV2O, and MV2O, respectively. The varieties and isolation methods affected these yields significantly $(\mathrm{p}<0.05)$. Furthermore, the bigger rhizomes, SV2O and $\mathrm{MV} 2 \mathrm{O}$, produced higher yields than the smaller counterparts, while maceration gave higher yields than the steam distillation. The study reports higher SV1O and $\mathrm{SV} 2 \mathrm{O}$ oil yields than the previous study from Indian counterparts (1.30\%) [6] and (3.50\%) [11]. The differences are possibly due to physiological variations as well as environmental conditions. Similarly, MV1O and $\mathrm{MV} 2 \mathrm{O}$ showed variations yield, with extraction by maceration technique, using $n$-hexane solvent. These yields were higher than those extracted using ethanol 95\% (3.21-5.98\%) as reported by Huda et al. [19].

Specific gravity is the ratio of a substance's density, respective to the density of water. Generally, essential oils have specific gravity values below 1 , except for a few containing oxygenated aromatic compounds. In this study, MV1O and MV2O have higher specific gravity than SV1O and SV2O from the two rhizome varieties. MV1O has the highest value of $0.90 \pm 0.01 \mathrm{~g} / \mathrm{mL}$, followed by MV2O $(0.88 \pm 0.01)$, SV1O $(0.86 \pm 0.01)$, and SV2O $(0.83 \pm 0.01)$, at $30^{\circ} \mathrm{C}$. These findings agreed with the values obtained for the essential oils of $K$. galanga rhizomes from Java $(0.87-0.88 \mathrm{~g} / \mathrm{mL})$. However, the results are inconsistent with the report by Srivastava et al. [6],

Table 1. Yields and physicochemical properties of $K$. galanga, L rhizome oil

\begin{tabular}{lllll}
\hline Properties & SV1O & MV1O & SV2O & MV2O \\
\hline Yield $(\%)$ & $2.81 \pm 0.09$ & $7.93 \pm 0.20$ & $3.60 \pm 0.10$ & $8.76 \pm 0.22$ \\
Physical state & Transparent liquid & Viscous & Transparent liquid & Viscous \\
Color & Light yellow & Yellow & Light yellow & Yellow \\
Odor & Aromatic & Aromatic & Aromatic & Aromatic \\
Solubility in water & Immiscible & Immiscible & Immiscible & Immiscible \\
Solubility in hexane & Miscible & Miscible & Miscible & Miscible \\
Solubility in ethanol & Miscible & Miscible & Miscible & Miscible \\
Specific density $(\mathrm{g} / \mathrm{mL})$ at $30^{\circ} \mathrm{C}$ & $0.86 \pm 0.01$ & $0.90 \pm 0.01$ & $0.83 \pm 0.01$ & $0.88 \pm 0.01$ \\
Refractive index at $30^{\circ} \mathrm{C}$ & $1.48 \pm 0.00$ & $1.49 \pm 0.00$ & $1.48 \pm 0.00$ & $1.49 \pm 0.00$ \\
Optical rotation at $30^{\circ} \mathrm{C}$ & -2.45 & -2.56 & -3.26 & -3.31 \\
Acid value $(\mathrm{mg} \mathrm{KOH} / \mathrm{g})$ & $1.23 \pm 0.01$ & $1.35 \pm 0.01$ & $1.82 \pm 0.02$ & $1.98 \pm 0.01$ \\
Saponification value $(\mathrm{mg} \mathrm{KOH} / \mathrm{g})$ & $103.50 \pm 0.67$ & $104.73 \pm 0.84$ & $106.85 \pm 1.02$ & $107.56 \pm 0.69$ \\
Ester value $(\mathrm{mg} \mathrm{KOH} / \mathrm{g})$ & $179.75 \pm 3.35$ & $180.55 \pm 3.30$ & $190.25 \pm 5.10$ & $191.35 \pm 4.20$ \\
Iodine value $(\mathrm{g} / \mathrm{l00 \textrm {g }})$ & $97 \pm 0.20$ & $99 \pm 0.21$ & $105 \pm 0.35$ & $104 \pm 0.33$ \\
\hline
\end{tabular}

I Wayan Muderawan et al. 
showing the essential oil of $K$. galanga rhizome from India isolated by hydrodistillation has a specific gravity of 1.03 $\mathrm{g} / \mathrm{mL}$, at $25^{\circ} \mathrm{C}$.

Based on the table above, SV2O and MV2O were discovered to have higher optical rotation $\left(-3.26^{\circ}\right.$ and $\left.3.31^{\circ}\right)$, compared to SV1O and $\mathrm{MV} 1 \mathrm{O}\left(-2.45^{\circ}\right.$ and $\left.-2.56^{\circ}\right)$. The oils isolated by maceration with $n$-hexane (MV1O and MV2O) also have higher optical activity than the steam distillation counterparts. Furthermore, the study samples have similar refractive index values, ranging from 1.4771 to 1.4855 , indicating the samples are rich in terpenes and oxygenated terpenes.

The acid value measures the amount of acids present in the oil and is expressed as a number of $\mathrm{KOH}$ milligrams required to neutralize the acid present in one gram of oil. This method is an indirect method for determining the acid amount in oil samples and, consequently, the oil's edibility. The acid values (AV) recorded in this study were $1.23 \pm 0.01, \quad 1.35 \pm 0.01, \quad 1.82 \pm 0.02$, and $1.98 \pm 0.01 \mathrm{mg}$ $\mathrm{KOH} / \mathrm{g}$, for SV1O, MV1O, SV2O, and $\mathrm{MV} 2 \mathrm{O}$, respectively. The acid value was significantly $(p<0.05)$ different for all oils and affected by varieties and isolation methods. These values are higher, compared to the results reported for Indian rhizome counterparts by Kumar (1.12 $\mathrm{mg} \mathrm{KOH} / \mathrm{g}$ ) [15], but lower, compared to the report by Srivastava et al. (2.24 mg KOH/g) [6]. The lower the acid value, the better the oil quality.

Meanwhile, the number of $\mathrm{KOH}$ milligrams required to saponify one gram of oil completely is called saponification value (SV). In this study, the SVs were greater for oils from smaller K. galanga rhizomes than the bigger counterparts and were significantly different $(p<$ $0.05)$. The saponification values were found to be $103.50 \pm 0.67,104.73 \pm 0.84,106.85 \pm 1.02$, and $107.56 \pm 0.69$ $\mathrm{mg} \mathrm{KOH} / \mathrm{g}$, for $\mathrm{SV} 1 \mathrm{O}, \mathrm{MV} 1 \mathrm{O}, \mathrm{SV} 2 \mathrm{O}$, and $\mathrm{MV} 2 \mathrm{O}$, respectively. The values were lower, compared to the report by Kumar (190.7 mg KOH/g) [15], and had similar values with the report by Srivastava et al. (106.59 mg $\mathrm{KOH} / \mathrm{g}$ ) [6]. The lower the saponification value, the better the oil quality.

The ester values (EV) were high in all oils and found $179.75 \pm 3.35,180.55 \pm 3.30,190.25 \pm 5.10$, and $191.35 \pm 4.20$ $\mathrm{mg} \mathrm{KOH} / \mathrm{g}$ for SV1O, MV1O, SV2O, and MV2O, respectively, that probably due to the high content of cinnamic esters in oils. The values were comparable with the results reported by Kumar (189.65 mg KOH/g) [15]. In addition, the EVs were found to be greater for oils from smaller $K$. galanga rhizomes than the bigger counterparts and were significantly $(p<0.05)$ different.

Iodine value (IV) refers to the number of iodine grams absorbed per 100 grams of fat or oil. This qualitative parameter denotes the oil sample's degree of unsaturation. The high iodine value of oils indicates the high content of unsaturation and the high quality of the oil. In this study, the samples had IVs of $97 \pm 0.20$, $99 \pm 0.21,105 \pm 0.35$, and $104 \pm 0.33 \mathrm{~g} / 100 \mathrm{~g}$ of oil for SV1O, MV1O, SV2O, and MV2O, respectively. The value was significantly $(p<0.05)$ different for all oils and affected by varieties and isolation methods. These values were slightly lower than a previous report $(107 \mathrm{~g} / 100 \mathrm{~g}$ oil) [6].

\section{Chemical Constituent of the Oils}

Fig. 2 shows the GC-MS chromatograms of four oils, SV1O, MV1O, SV2O, and MV2O, from two K. galanga rhizome varieties isolated by steam distillation and maceration. Meanwhile, Table 2 shows the oils' chemical constituents, determined based on mass fragmentation patterns and comparison with mass spectra of Wiley library.

The total number of compounds identified in oils isolated by steam distillation and maceration from both K. galanga rhizome varieties were 48 (SV1O), 46 (MV1O), 59 (SV2O), and 53 (MV2O), representing $99.88,99.95,99.94$, and $99.95 \%$ of the total oil, respectively. These show that the chemical components of oils from both varieties differ significantly in number and quantities. However, oils' chemical constituents from the isolation methods, steam distillation, and maceration were almost identical, which means the chemical constituents of oils from K. galanga rhizomes cultivated in Indonesia strongly depend on varieties rather than isolation methods. The oils contain monoterpenes, oxygenated monoterpenes, sesquiterpenes, oxygenated sesquiterpenes, long-chain alkane hydrocarbons, compounds derived from shikimic 


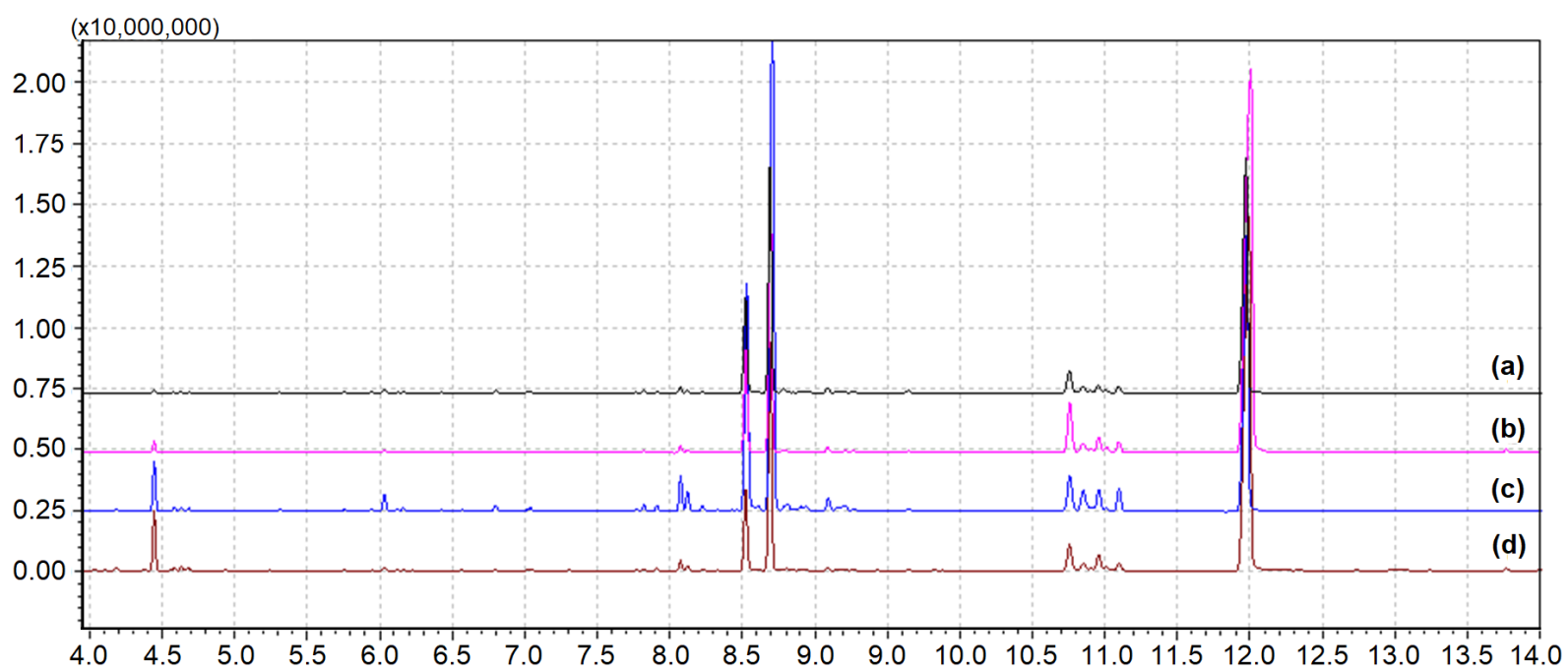

Fig 2. An overlay of K. galanga, L. rhizome oils chromatograms. (a) SV1O, (b) MV1O, (c) SV2O, and (d) MV2O

Table 2. Chemical composition of K. galanga, L. rhizome oils

\begin{tabular}{|c|c|c|c|c|c|c|c|c|}
\hline \multirow{2}{*}{ No } & \multirow{2}{*}{$\mathrm{RT}$} & \multirow{2}{*}{ Common Name } & \multirow{2}{*}{$\begin{array}{l}\text { Molecular } \\
\text { Formula }\end{array}$} & \multirow{2}{*}{$\begin{array}{l}\text { MW } \\
(\mathrm{g} / \mathrm{mol})\end{array}$} & \multicolumn{4}{|c|}{$\%$ Area } \\
\hline & & & & & SV1O & MV1O & SV2O & $\mathrm{MV} 2 \mathrm{O}$ \\
\hline 1 & 3.629 & (-)- $\alpha$-Pinene & $\mathrm{C}_{10} \mathrm{H}_{16}$ & 136.23 & - & 0.02 & 0.09 & 0.25 \\
\hline 2 & 3.806 & Camphene & $\mathrm{C}_{10} \mathrm{H}_{16}$ & 136.23 & - & 0.04 & 0.13 & 0.30 \\
\hline 3 & 3.928 & Benzaldehyde & $\mathrm{C}_{7} \mathrm{H}_{6} \mathrm{O}$ & 106.12 & - & - & 0.02 & - \\
\hline 4 & 4.108 & (-)- $\beta$-Pinene & $\mathrm{C}_{10} \mathrm{H}_{16}$ & 136.23 & - & - & - & 0.05 \\
\hline 5 & 4.185 & 7-Methyl-3-methylene-1,6-octadiene & $\mathrm{C}_{10} \mathrm{H}_{16}$ & 136.23 & - & 0.03 & 0.15 & 0.23 \\
\hline 6 & 4.377 & (-)-Phelandrene & $\mathrm{C}_{10} \mathrm{H}_{16}$ & 136.23 & - & - & 0.06 & 0.08 \\
\hline 7 & 4.445 & $\Delta$-3-Carene & $\mathrm{C}_{10} \mathrm{H}_{16}$ & 136.23 & 0.22 & 0.64 & 2.34 & 3.97 \\
\hline 8 & 4.582 & 1-Methyl-3-(1-methylethyl)-benzene & $\mathrm{C}_{10} \mathrm{H}_{14}$ & 134.22 & 0.04 & 0.05 & - & - \\
\hline 9 & 4.586 & 1-Methyl-4-(1-methylethyl)-benzene & $\mathrm{C}_{10} \mathrm{H}_{14}$ & 134.22 & - & - & 0.22 & 0.28 \\
\hline 10 & 4.632 & (-)-Limonene & $\mathrm{C}_{10} \mathrm{H}_{16}$ & 136.23 & 0.07 & 0.05 & 0.22 & 0.27 \\
\hline 11 & 4.687 & 1,8-Cineole & $\mathrm{C}_{10} \mathrm{H}_{18} \mathrm{O}$ & 154.25 & 0.04 & 0.04 & 0.16 & 0.18 \\
\hline 12 & 4.937 & $\gamma$-Terpinene & $\mathrm{C}_{10} \mathrm{H}_{16}$ & 136.23 & - & - & 0.03 & 0.03 \\
\hline 13 & 5.245 & $\alpha$-Terpinolene & $\mathrm{C}_{10} \mathrm{H}_{16}$ & 136.23 & - & - & 0.05 & 0.04 \\
\hline 14 & 5.313 & (E)-Farnesene epoxide & $\mathrm{C}_{15} \mathrm{H}_{24} \mathrm{O}$ & 220.35 & 0.04 & - & 0.07 & 0.03 \\
\hline 15 & 5.758 & Ipsdienol & $\mathrm{C}_{10} \mathrm{H}_{16} \mathrm{O}$ & 152.23 & 0.08 & 0.03 & 0.09 & 0.04 \\
\hline 16 & 5.946 & p-Mentha-1,5-dien-8-ol & $\mathrm{C}_{10} \mathrm{H}_{16} \mathrm{O}$ & 152.23 & 0.08 & - & 0.14 & 0.03 \\
\hline 17 & 6.033 & endo-Borneol & $\mathrm{C}_{10} \mathrm{H}_{18} \mathrm{O}$ & 154.25 & 0.43 & 0.15 & 0.87 & 0.27 \\
\hline 18 & 6.122 & 4-Acetoxy-tricyclo[4.3.1.0(3,8)]-dec-10-yl acette & $\mathrm{C}_{14} \mathrm{H}_{20} \mathrm{O}_{4}$ & 252.31 & 0.10 & - & - & - \\
\hline 19 & 6.162 & $\alpha, \alpha, 4$-Trimethyl-benzenemethanol & $\mathrm{C}_{10} \mathrm{H}_{14} \mathrm{O}$ & 150.22 & 0.09 & 0.04 & 0.18 & 0.06 \\
\hline 20 & 6.229 & $\beta$-Fenchyl alcohol & $\mathrm{C}_{10} \mathrm{H}_{18} \mathrm{O}$ & 154.25 & 0.04 & - & 0.05 & 0.03 \\
\hline 21 & 6.424 & Eucarvone & $\mathrm{C}_{10} \mathrm{H}_{14} \mathrm{O}$ & 150.22 & 0.06 & - & 0.09 & - \\
\hline 22 & 6.566 & 2-Methyl-6-methylene-2,7-octadien-4-ol & $\mathrm{C}_{10} \mathrm{H}_{16} \mathrm{O}$ & 152.23 & - & - & 0.06 & 0.03 \\
\hline 23 & 6.799 & 4-Methoxy benzaldehyde & $\mathrm{C}_{8} \mathrm{H}_{8} \mathrm{O}_{2}$ & 136.15 & 0.25 & 0.04 & 0.32 & 0.06 \\
\hline 24 & 7.040 & Tridecane & $\mathrm{C}_{13} \mathrm{H}_{28}$ & 184.36 & 0.20 & 0.12 & 0.29 & 0.13 \\
\hline 25 & 7.304 & Verbenone & $\mathrm{C}_{10} \mathrm{H}_{14} \mathrm{O}$ & 150.22 & - & - & 0.07 & 0.08 \\
\hline 26 & 7.373 & Cyclofenchene & $\mathrm{C}_{10} \mathrm{H}_{16}$ & 136.23 & - & - & 0.03 & - \\
\hline 27 & 7.560 & $\alpha$-Cubebene & $\mathrm{C}_{15} \mathrm{H}_{24}$ & 204.35 & - & - & 0.02 & - \\
\hline 28 & 7.768 & $\alpha$-Ylangene & $\mathrm{C}_{15} \mathrm{H}_{24}$ & 204.35 & 0.12 & 0.05 & 0.19 & 0.05 \\
\hline
\end{tabular}

I Wayan Muderawan et al. 
Table 2. Chemical composition of K. galanga, L. rhizome oils (Continued)

\begin{tabular}{|c|c|c|c|c|c|c|c|c|}
\hline \multirow{2}{*}{ No } & \multirow{2}{*}{ RT } & \multirow{2}{*}{ Common Name } & \multirow{2}{*}{$\begin{array}{l}\text { Molecular } \\
\text { Formula }\end{array}$} & \multirow{2}{*}{$\begin{array}{l}\text { MW } \\
(\mathrm{g} / \mathrm{mol})\end{array}$} & \multicolumn{4}{|c|}{$\%$ Area } \\
\hline & & & & & SV1O & MV1O & SV2O & MV2O \\
\hline 29 & 7.822 & Tetradecane & $\mathrm{C}_{14} \mathrm{H}_{30}$ & 198.39 & 0.25 & 0.13 & 0.35 & 0.12 \\
\hline 30 & 7.911 & $(-)$ - $\beta$-Elemene & $\mathrm{C}_{15} \mathrm{H}_{24}$ & 204.35 & 0.12 & 0.06 & 0.31 & 0.15 \\
\hline 31 & 8.074 & $\beta$-Patchoulene & $\mathrm{C}_{15} \mathrm{H}_{24}$ & 204.35 & 0.70 & 0.48 & 1.87 & 0.76 \\
\hline 32 & 8.121 & $(+)$ - $\alpha$-Gurjunene & $\mathrm{C}_{15} \mathrm{H}_{24}$ & 204.35 & 0.30 & 0.21 & 1.02 & 0.41 \\
\hline 33 & 8.224 & trans-Caryophyllene & $\mathrm{C}_{15} \mathrm{H}_{24}$ & 204.35 & 0.14 & 0.06 & 0.35 & 0.11 \\
\hline 34 & 8.327 & $\alpha$-Guaiene & $\mathrm{C}_{15} \mathrm{H}_{24}$ & 204.35 & 0.13 & 0.03 & 0.14 & 0.08 \\
\hline 35 & 8.380 & (-)-Aristolene & $\mathrm{C}_{15} \mathrm{H}_{24}$ & 204.35 & 0.06 & - & 0.11 & - \\
\hline 36 & 8.432 & Selina-3,7(11)-diene & $\mathrm{C}_{15} \mathrm{H}_{24}$ & 204.35 & - & - & 0.20 & - \\
\hline 37 & 8.524 & Ethyl trans-cinnamate & $\mathrm{C}_{11} \mathrm{H}_{12} \mathrm{O}_{2}$ & 176.21 & 12.54 & 7.06 & 14.44 & 6.97 \\
\hline 38 & 8.610 & $(+)$-Sativen & $\mathrm{C}_{15} \mathrm{H}_{24}$ & 204.30 & - & 0.09 & 0.38 & 0.13 \\
\hline 39 & 8.607 & $\alpha$-Amorphene & $\mathrm{C}_{15} \mathrm{H}_{24}$ & 204.35 & 0.20 & - & - & - \\
\hline 40 & 8.696 & Pentadecane & $\mathrm{C}_{16} \mathrm{H}_{34}$ & 226.44 & 28.67 & 19.16 & 37.21 & 18.78 \\
\hline 41 & 8.785 & Germacrene D & $\mathrm{C}_{15} \mathrm{H}_{24}$ & 204.35 & 0.73 & 0.32 & - & - \\
\hline 42 & 8.810 & $\begin{array}{l}\text { 2-Isopropenyl-4a,8-dimethyl-1,2,3,4,4a,5,6,7- } \\
\text { octahydro-naphthalene }\end{array}$ & $\mathrm{C}_{15} \mathrm{H}_{24}$ & 204.35 & - & - & 0.77 & 0.31 \\
\hline 43 & 8.855 & $(-)-\beta$-Silnene & $\mathrm{C}_{15} \mathrm{H}_{24}$ & 204.35 & 0.08 & 0.04 & 0.16 & 0.05 \\
\hline 44 & 8.898 & $(+)$-Valencene & $\mathrm{C}_{15} \mathrm{H}_{24}$ & 204.35 & 0.16 & 0.07 & 0.34 & 0.11 \\
\hline 45 & 8.935 & $\alpha$-Salinene & $\mathrm{C}_{15} \mathrm{H}_{24}$ & 204.35 & 0.16 & 0.07 & - & - \\
\hline 46 & 8.938 & $\beta$-Chamigrene & $\mathrm{C}_{15} \mathrm{H}_{24}$ & 204.35 & - & - & 0.36 & - \\
\hline 47 & 8.965 & 2,6-Bis(1,1-dimethylethyl)-4-methyl-phenol & $\mathrm{C}_{15} \mathrm{H}_{24} \mathrm{O}$ & 220.30 & 0.08 & - & - & - \\
\hline 48 & 9.003 & $(-)$-Sinularene & $\mathrm{C}_{15} \mathrm{H}_{24}$ & 204.35 & - & - & 0.06 & - \\
\hline 49 & 9.090 & $(-)-\gamma$-Cadinene & $\mathrm{C}_{15} \mathrm{H}_{24}$ & 204.35 & 0.73 & 0.41 & 0.82 & 0.30 \\
\hline 50 & 9.152 & $(+)$ - $\Delta$-Cadinene & $\mathrm{C}_{15} \mathrm{H}_{24}$ & 204.35 & 0.19 & 0.08 & 0.33 & \\
\hline 51 & 9.155 & (-)-Valencene & $\mathrm{C}_{15} \mathrm{H}_{24}$ & 204.35 & - & - & - & 0.10 \\
\hline 52 & 9.206 & $(+)-\gamma$-Gurjunene & $\mathrm{C}_{15} \mathrm{H}_{24}$ & 204.35 & 0.27 & 0.17 & - & 0.21 \\
\hline 53 & 9.210 & $\Delta$-Guaiene & $\mathrm{C}_{15} \mathrm{H}_{24}$ & 204.35 & - & - & 0.57 & - \\
\hline 54 & 9.267 & (-)-Isoledene & $\mathrm{C}_{15} \mathrm{H}_{24}$ & 204.35 & 0.27 & 0.20 & 0.17 & 0.16 \\
\hline 55 & 9.433 & Elemol & $\mathrm{C}_{15} \mathrm{H}_{26} \mathrm{O}$ & 222.37 & 0.04 & - & 0.06 & 0.03 \\
\hline 56 & 9.585 & (-)-Isoledene & $\mathrm{C}_{15} \mathrm{H}_{24}$ & 204.35 & 0.04 & - & - & - \\
\hline 57 & 9.649 & Germacrene B & $\mathrm{C}_{15} \mathrm{H}_{24}$ & 204.35 & 0.44 & 0.13 & 0.17 & 0.06 \\
\hline 58 & 9.755 & Hexadecane & $\mathrm{C}_{16} \mathrm{H}_{34}$ & 226.44 & - & - & 0.07 & - \\
\hline 59 & 9.882 & Spathulenol & $\mathrm{C}_{15} \mathrm{H}_{24} \mathrm{O}$ & 220.35 & 0.04 & - & 0.07 & - \\
\hline 60 & 9.984 & 2,4,6-Tris(1,1-dimethylethyl)-phenol & $\mathrm{C}_{18} \mathrm{H}_{30} \mathrm{O}$ & 262.40 & 0.07 & - & - & - \\
\hline 61 & 9.987 & (-)-Caryophyllene oxide & $\mathrm{C}_{15} \mathrm{H}_{24} \mathrm{O}$ & 220.35 & - & - & 0.09 & - \\
\hline 62 & 10.247 & Veridiflorol & $\mathrm{C}_{15} \mathrm{H}_{26} \mathrm{O}$ & 222.37 & - & - & 0.06 & - \\
\hline 63 & 10.333 & Cubenol & $\mathrm{C}_{15} \mathrm{H}_{26} \mathrm{O}$ & 222.37 & 0.07 & 0.04 & 0.08 & - \\
\hline 64 & 10.411 & Azulene & $\mathrm{C}_{10} \mathrm{H}_{8}$ & 128.17 & 0.06 & - & - & - \\
\hline 65 & 10.755 & Ethyl trans-m-methoxycinnamate & $\mathrm{C}_{12} \mathrm{H}_{14} \mathrm{O}_{3}$ & 206.24 & 3.87 & 4.53 & 2.92 & 2.90 \\
\hline 66 & 10.848 & (E)-9-Eicosene & $\mathrm{C}_{20} \mathrm{H}_{40}$ & 280.50 & 1.22 & 1.02 & 1.81 & 0.97 \\
\hline 67 & 10.905 & 7-Decen-2-one & $\mathrm{C}_{10} \mathrm{H}_{18} \mathrm{O}$ & 154.25 & 0.33 & - & 0.28 & - \\
\hline 68 & 10.906 & 1-Cyclododecyl-ethanone & $\mathrm{C}_{14} \mathrm{H}_{26} \mathrm{O}$ & 210.36 & - & 0.32 & - & 0.24 \\
\hline 69 & 10.956 & $(Z 6, Z 9)$-Pentadecadien-1-ol & $\mathrm{C}_{15} \mathrm{H}_{28} \mathrm{O}$ & 224.38 & 1.21 & 1.30 & 1.55 & 1.61 \\
\hline 70 & 11.008 & Methyl trans-p-methoxycinnamate & $\mathrm{C}_{11} \mathrm{H}_{12} \mathrm{O}_{3}$ & 192.21 & 0.50 & 0.53 & 0.24 & 0.36 \\
\hline 71 & 11.096 & Eicosane & $\mathrm{C}_{20} \mathrm{H}_{42}$ & 282.55 & 0.98 & 0.90 & 1.54 & 0.76 \\
\hline
\end{tabular}


Table 2. Chemical composition of $K$. galanga, L. rhizome oils (Continued)

\begin{tabular}{|c|c|c|c|c|c|c|c|c|}
\hline \multirow{2}{*}{ No } & \multirow{2}{*}{ RT } & \multirow{2}{*}{ Common Name } & \multirow{2}{*}{$\begin{array}{l}\text { Molecular } \\
\text { Formula }\end{array}$} & \multirow{2}{*}{$\begin{array}{l}\text { MW } \\
(\mathrm{g} / \mathrm{mol})\end{array}$} & \multicolumn{4}{|c|}{$\%$ Area } \\
\hline & & & & & SV1O & MV1O & SV2O & MV2O \\
\hline 72 & 11.988 & Ethyl trans- $p$-methoxycinnamate & $\mathrm{C}_{12} \mathrm{H}_{14} \mathrm{O}_{3}$ & 206.24 & 43.37 & 60.62 & 24.92 & 57.17 \\
\hline 73 & 12.737 & 4-(4-Methoxyphenyl)oxane-4-carboxylic acid & $\mathrm{C}_{13} \mathrm{H}_{16} \mathrm{O}_{4}$ & 236.26 & - & - & - & 0.04 \\
\hline 74 & 12.739 & Methyl trans-3,4- dimethoxycinnamate & $\mathrm{C}_{12} \mathrm{H}_{14} \mathrm{O}_{4}$ & 222.24 & - & 0.03 & - & - \\
\hline 75 & 12.960 & 9,12-Octadecadien-1-ol/(Z)-9,17-Octadecadienal & $\mathrm{C}_{18} \mathrm{H}_{34} \mathrm{O}$ & 266.50 & - & 0.06 & - & 0.02 \\
\hline 76 & 13.011 & 1-Heptadecanol & $\mathrm{C}_{17} \mathrm{H}_{36} \mathrm{O}$ & 256.50 & - & - & - & 0.03 \\
\hline 77 & 13.013 & (Z)-9-Octadecenal & $\mathrm{C}_{18} \mathrm{H}_{34} \mathrm{O}$ & 266.50 & - & 0.14 & - & - \\
\hline 78 & 13.648 & Hexadecanoic acid & $\mathrm{C}_{16} \mathrm{H}_{32} \mathrm{O}_{2}$ & 256.42 & - & 0.03 & - & - \\
\hline 79 & 13.766 & 2-Imino-3-(3-nitrophenyl)-tetrahydrothiazol-4-one & $\mathrm{C}_{9} \mathrm{H}_{7} \mathrm{~N}_{3} \mathrm{O}_{3} \mathrm{~S}$ & 237.20 & - & 0.21 & - & 0.17 \\
\hline 80 & 13.999 & Sandaracopimaradiene & $\mathrm{C}_{20} \mathrm{H}_{32}$ & 272.50 & - & 0.08 & - & 0.07 \\
\hline \multirow[t]{2}{*}{81} & 17.457 & Ergosterol & $\mathrm{C}_{28} \mathrm{H}_{44} \mathrm{O}$ & 396.65 & - & 0.04 & - & 0.08 \\
\hline & & Total & & & 99.88 & 99.95 & 99.94 & 99.95 \\
\hline
\end{tabular}

acid, and other miscellaneous components. Table 2 shows the oils' constituents in detail.

The air-dried rhizome oils of both $K$. galanga varieties, isolated by steam distillation and maceration, consisted of compounds derived from shikimic acid, 60.53\% (SV1O), 72.81\% (MV1O), 42.86\% (SV2O), and 67.46\% (MV2O), respectively with ethyl trans-pmethoxycinnamate $(43.37 \%$ in SV1O, 60.62\% in MV1O, $24.92 \%$ in $\mathrm{SV} 2 \mathrm{O}$, and $57.17 \%$ in $\mathrm{MV} 2 \mathrm{O}$ ) as the major components. Meanwhile, the total trans-cinnamic acid derivatives contents, including ethyl trans-pmethoxycinnamate, ethyl trans-cinnamate, ethyl trans$m$-methoxycinnamate, methyl trans- $p$ methoxycinnamate, and methyl trans- $m, p$ dimethoxycinnamate, were 59.78, 72.21, 42.28, and $67.04 \%$ in SV1O, MV1O, SV2O, and MV2O, respectively. In addition, ethyl trans-p-methoxycinnamate and ethyltrans-cinnamate show monoamine oxidase inhibiting and larvicidal effects [20]. Also, the cinnamate derivatives are responsible for the spicy aromatic odor [21].

The higher alkane hydrocarbons with a percentage contribution of $31.32 \%$ in SV1O, $21.33 \%$ in MV1O, $39.46 \%$ in SV2O, and $19.79 \%$ in MV2O, were the second major constituent compound in oils from bigger $K$. galanga rhizome varieties, isolated by steam distillation and maceration. This result shows that steam distillation obtains more alkane hydrocarbons compared to maceration. In these oils, pentadecane $(28.67 \%$ in SV1O, $19.16 \%$ in MV1O, $37.21 \%$ in SV2O, and $18.78 \%$ MV2O) was the main higher alkane hydrocarbons detected. Meanwhile, the sesquiterpene hydrocarbons and oxygenated sesquiterpenes contributed 5.11, 2.51, 8.83, and $3.22 \%$, respectively, as the oil's third major constituents. Similarly, the steam distillation method obtained more sesquiterpenes and oxygenated sesquiterpenes content than the maceration method. In addition, the important sesquiterpenes and oxygenated sesquiterpenes identified in all oil samples (SV1O, MV1O, SV2O and MV2O) were $\alpha$-ylangene $(0.12,0.05$, $0.19,0.05 \%),(-)$ - $\beta$-elemene $(0.12,0.06,0.31$, and $0.15 \%)$, $\beta$-patchoulene $(0.70,0.48,1.87$, and $0.76 \%), \alpha$ gurjunene $(0.30,0.21,1.02,0.41 \%)$, trans-caryophyllene $(0.14,0.06,0.35$, and $0.11 \%)$, and $\alpha$-guaiene $(0.13,0.03$, 0.14 , and $0.08 \%$ ).

The essential oils from smaller rhizomes were discovered to have higher and oxygenated monoterpene contents (4.81 and 5.95\%), compared to oils from bigger rhizomes (1.15 and 1.09\%), isolated by both methods. Furthermore, the important and oxygenated monoterpenes present in all oil samples (SV1O, MV1O, SV2O and MV2O) were $\Delta$-3-carene $(0.22,0.64,2.34$ and 3.97\%), (-)-limonene (0.07, 0.05, 0.22 and $0.27 \%), 1,8$ cineole $(0.04,0.04,0.16$, and $0.18 \%)$, ipsdienol $(0.08$, $0.03,0.09$, and $0.04 \%)$ and endo-borneol $(0.43,0.15,0.87$, and $0.27 \%)$.

Fig. 3 shows the $K$. galanga rhizome oils also contained three saturated- and unsaturated long-chain alcohols (6Z,9Z-pentadeca-6,9-dien-1-ol, 9E,12E- 
octadeca-9,12-dien-1-ol, and heptadecan-1-ol), one longchain aldehyde ( $Z$-octadec- 9 -enal), as well as two longchain carboxylic acids (4-(4-methoxyphenyl)oxane-4carboxylic acid, hexadecanoic acid). It is interesting to note that the rhizome oils from both K. galanga varieties isolated by maceration were found to contain one diterpene compound (sandaracopimaradiene), one steroid (ergosterol), and one alkaloid (2-imino-3-(3nitrophenyl)-1,3-thiazolidin-4-one), in small quantities, Fig. 3. No other studies have never reported this finding. Meanwhile, sandaracopimaradiene is a diterpene derived from pimarane by dehydrogenation across the $\mathrm{C}(8)$ $\mathrm{C}(14)$ and $\mathrm{C}(15)-\mathrm{C}(16)$ bonds with a role as a metabolite and derived from an isopimarane hydride [21-22]. Furthermore, ergosterol is a phytosterol consisting of ergostane, with double bonds at the 5,6-, 7,8- and 22,23positions and a 3-beta-hydroxy group. The compound has a role as a fungal metabolite and a Saccharomyces cerevisiae metabolite [23] and is also a biological precursor of vitamin $\mathrm{D}_{2}$, called ergocalciferol. Exposure to ultraviolet light causes a photochemical reaction, converting ergosterol to ergocalciferol [24]. The essential oils were also contained some miscellaneous compounds as minor components.
Based on the results, numerous previously unreported compounds were not only identified in the rhizome oils from K. galanga, but the two varieties were also found to differ remarkably. The oils from variety- 2 (the plant with smaller rhizomes) contained more compounds than variety-1 (the plant with bigger rhizomes). A previous study reported only eight components identified in the essential oil of $K$. galanga rhizome from East Java, Indonesia, isolated by maceration using methanol as solvent [25]. Meanwhile, twenty five different compounds in K. galanga essential oil, representing $95.98 \%$ of the total oil were identified by Yang et al. [26] and fourty one components by Li et al. [27] in Chinese samples. In addition, Wong et al. [20] successfully identified 53 compounds in a Malaysian sample, with 34 compounds identified through the OV101 column and 19 through Carbowax 20M column, including indole, vanillin, and other compounds. Raina et al. [28] reported over 38 constituents in oil from South Indian samples, while Srivastava et al. [29] reported fifty four constituents identified from Hamirpur India which amounting to $92.77 \%$ of the Kaempferia galanga rhizome volatile organic compounds (VOCs). VOCs were ethyl trans-p-methoxycinnamate (52.54\%), ethyl

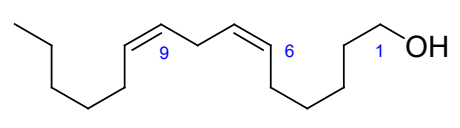

(6Z,9Z)-Pentadeca-6,9-dien-1-ol

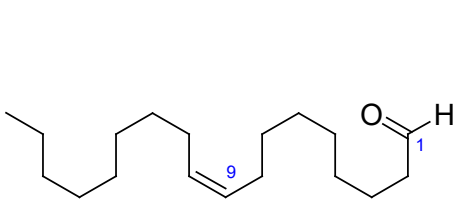

(Z)-Octadec-9-enal

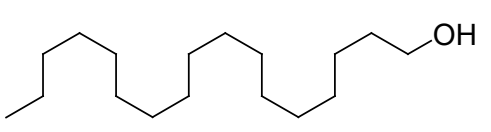

Heptadecan-1-ol

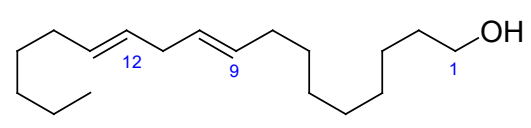

(9E,12E)-Octadeca-9,12-dien-1-ol

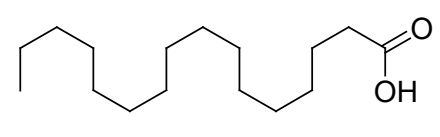

Hexadecanoic acid

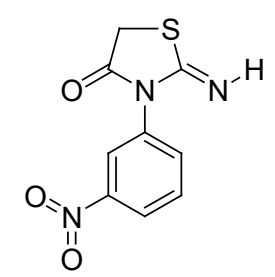

2-Imino-3-(3-nitrophenyl)tetrahydrothiazol-4-one
4-(4-Methoxyphenyl)oxane-4-carboxylic acid
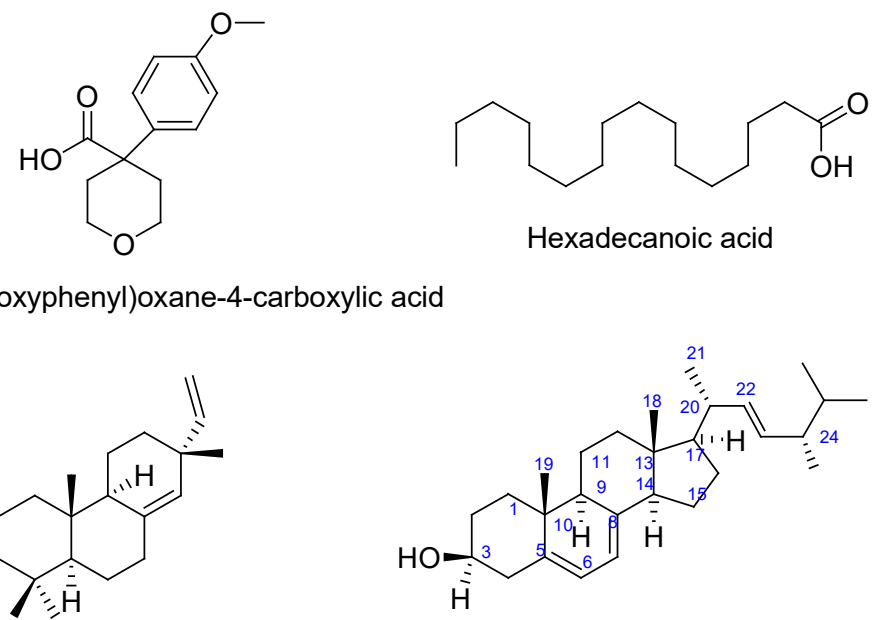

Sandaracopimaradiene

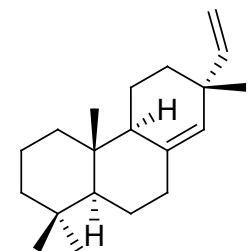

(22E,24S)-24-Methyl-27-norcholesta-5,7,22trien-3- -ol (ergosterol)

Fig 3. Structures of selected metabolites identified in the K. galanga rhizome oils 
trans-cinnamate (24.98\%), 1,8-cineole (4.14\%), 3-carene (3.94\%), dihydroterpineol (1.84\%), a-terpineol (1.64\%), and camphene $(1.02 \%)$.

This study identified 48 and 46 compounds in the bigger $K$. galanga rhizome oils isolated by steam distillation and maceration. The two oils contained 36 similar compounds. However, 12 compounds were identified in SV1O but not in MV1O. Meanwhile, 59 and 53 compounds were identified in the smaller rhizome oils, isolated by steam distillation and maceration, respectively. However, 43 compounds were common to both oils, while 16 were present in SV2O but not in MV2O. A total of 81 compounds were identified in all oils, with 28 similar compounds, including ethyl trans-pmethoxycinnamate (24.92-60.64\%), ethyl trans-cinnamate (6.97-14.44\%), ethyl trans-m-methoxycinnamate (2.90$4.53 \%)$ and methyl trans-p-methoxycinnamate (0.24$0.53 \%)$. Other important chemical compounds identified in all oil samples were $\delta$-3-carene $(0.22-3.97 \%)$, (-)limonene $(0.05-0.27 \%)$, 1,8-cineole $(0.04-0.18 \%)$, borneol (0.15-0.87\%), and pentadecane (18.78-37.21\%).

As seen in Fig. 4, the analyzed rhizome essential oils from two K. galanga varieties isolated by steam distillation and maceration contained major compounds derived from shikimic acid, phenylpropanoids, with contributions of $42.86-72.81 \%$, followed by higher alkanes hydrocarbon (19.79-39.46\%), sesquiterpenes hydrocarbons (2.47-8.34\%), miscellaneous compounds (1.89-4.04\%), monoterpenes hydrocarbons (0.76$5.49 \%)$, oxygenated monoterpenes $(0.11-0.84 \%)$, and oxygenated sesquiterpenes (0.04-0.43\%). Furthermore, ethyl trans-p-methoxycinnamate (24.92-60.62\%) was discovered to be the most prevalent chemical compound derived from shikimic acid in oils.

The quantitative data, especially on the main chemical component of $K$. galanga oils tested, were quite comparable with the counterparts reported in the literature from other regions of the world. However, in some cases, a notable variation in the oils' composition was also observed, which agrees with previous studies stating a considerable variation in the rhizome oils' main chemical composition (18.42-63.36\%) for varieties and isolation methods. Wong et al. [20] reported the constituent of steam-distilled essential oil from fresh $K$. galanga, L. rhizomes growing in Malaysia to be ethyl trans-p-methoxycinnamate $(51.6 \%)$, ethyl cinnamate (16.5\%), pentadecane (9.0\%), 1,8-cineole (5.7\%), 6-car3 -ene (3.3\%) and borneol (2.7\%). Meanwhile, Baharudin et al. [30] identified ethyl trans-p-methoxycinnamate (57.2\%) and ethyl cinnamate $(39.1 \%)$ as the major constituents of essential oils of K. galanga from Pahang, Malaysia. In addition, the chemical components of volatile

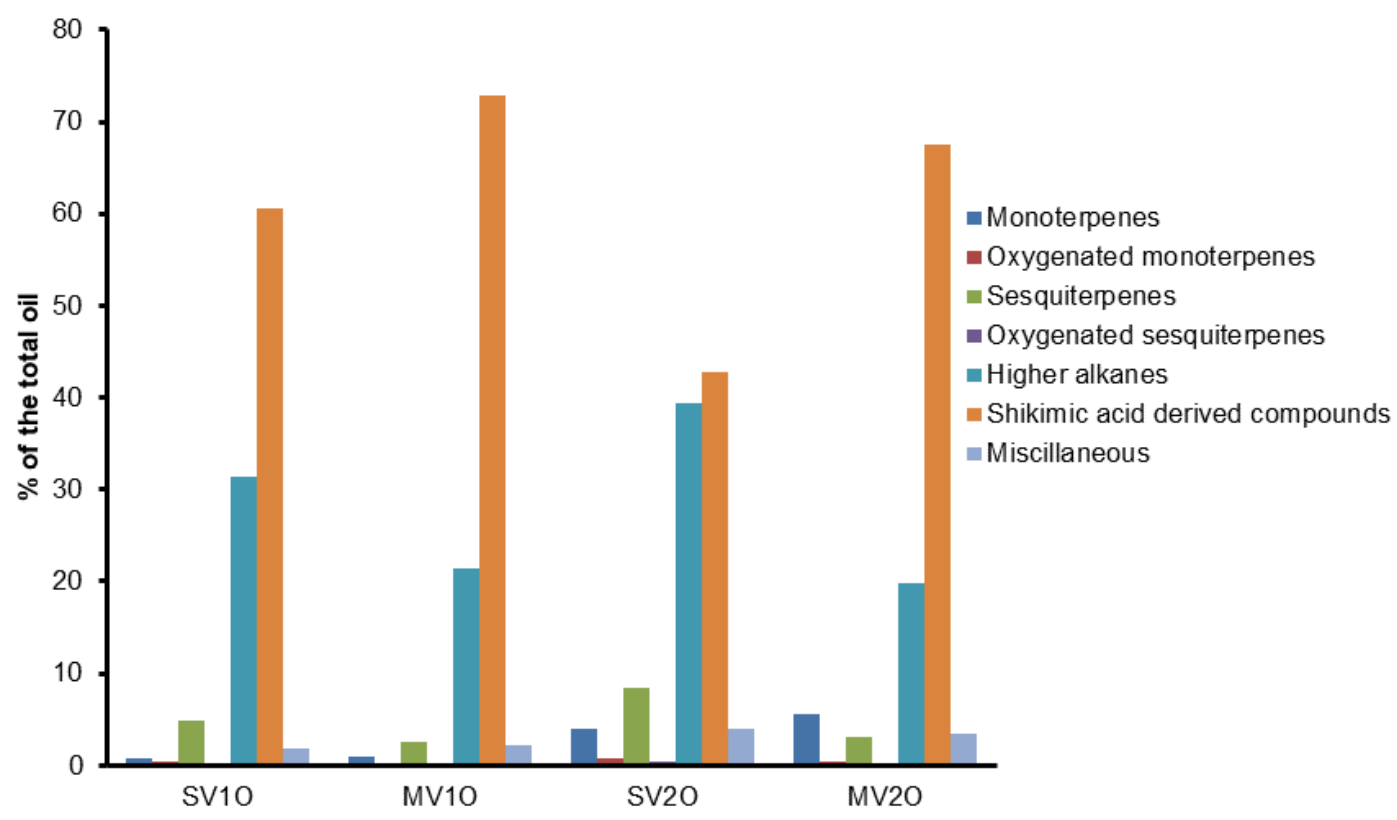

Fig 4. The proportion of different compound classes in K. galanga rhizome essential oils 
K. galanga dried rhizome oil from Amphur Chana, Songkhla Province, Thailand, obtained by steam distillation, were reported to be ethyl trans- $p$ methoxycinnamate $(31.77 \%)$, methyl trans-cinnamate (23.23\%), carvone (11.13\%), eucalyptol (9.59\%), pentadecane $(6.41 \%)$, borneol (2.87\%), camphene (2.24\%), benzene (1.33\%), and pinene (1.23\%) [31]. A separate study reported that essential oil from $K$. galanga obtained from Chiang Mai province, Thailand, isolated by steam distillation, contained ethyl trans-pmethoxycinnamate (25.96\%), pentadecane $(26.10 \%)$, and eucalyptol (2.12\%) [32]. Meanwhile, the Bangladesh counterpart obtained by hydrodistillation was reported to contain ethyl trans-p-methoxycinnamate (63.36\%), ethyl cinnamate (6.31\%), 4-cyclooctene-1-methanol (4.61\%), caryophyllene oxide (4.37\%), and limonene (3.22\%) [33]. Also, the essential oils from two K. galanga Linn varieties, 'Kasthuri' and 'Rajani' from India, were reported to contain ethyl trans-p-methoxycinnamate (39 and 35\%, respectively) [29]. In addition, essential oil from dried Kaempferia galanga L. rhizome, from Herbal Garden Rishikesh, India, isolated by steam distillation, was reported to contain ethyl trans-cinnamate (29.48\%), ethyl trans-p-methoxycinnamate $\quad(18.42 \%), \quad \gamma$-cadinene (9.81\%), 1,8-cineole (6.54\%), $\delta$-carene (6.19\%), borneol (5.21\%), ethyl trans-m-methoxycinnamate (2.15\%), camphene (1.58\%), linoleoyl chloride (1.35\%), and $\alpha$ pinene (1.32\%) [15].

In this study, the analyzed air-dried rhizome essential oils, isolated by steam distillation and maceration, using $n$-hexane as the solvent, contained mainly ethyl trans-p-methoxycinnamate, although significantly varying concentration, compared to other $K$. galanga varieties and isolation methods employed. The highest oil yield was obtained from smaller rhizome varieties isolated by maceration (MV2O), while the highest ethyl trans-pmethoxycinnamate content was obtained from bigger counterparts isolated by maceration (MV1O).

\section{Antioxidant Activities}

Antioxidants can either delay or inhibit oxidation processes occurring under atmospheric oxygen or reactive oxygen species. Thus, these compounds are involved in an organism's defense mechanism against pathologies associated with free radical attacks. In the Zingiberaceae family, antioxidants produced by the plant are generally believed to be transported and accumulated in the rhizomes, implying that rhizomes are bound to have higher antioxidant activity than other plant parts. In this present study, antioxidant activities of four oils from two K. galanga rhizome varieties were assessed for comparison purposes, and all samples exhibited high antioxidant activities. The antioxidant activity in DPPH free radical-scavenging assay of all oil samples expressed as $\mathrm{IC}_{50}$ values were found to be $86.10 \pm 1.51,85.24 \pm 1.48,89.19 \pm 1.72$, and $86.49 \pm 2.03$ $\mu \mathrm{g} / \mathrm{mL}$, for SV1O, MV1O, SV2O, and MV2O, respectively. These values did not differ significantly ( $\mathrm{p}>0.05$ ), which means the K. galanga rhizome oils' antioxidant activities are high and not dependent on the variety and isolation method.

Chan et al. [4] reported weak antioxidant activity exhibited by methanol extract of $K$. galanga from Selangor Malaysia. According to the study, the antioxidant activity expressed as ascorbic acid equivalent (AAE) was $17 \pm 1 \mathrm{mg} \mathrm{AA} / 100 \mathrm{~g}$ for the rhizomes. However, Ali et al. [18] reported high antioxidant activity exhibited by methanolic extract of K. galanga plant. The antioxidant activity represented as $\mathrm{IC}_{50}$ of DPPH radical scavenging activity of $K$. galanga plant from Chittagong Bangladesh dried in an air was $16.58 \mu \mathrm{g} / \mathrm{mL}$. Sahoo et al. also evaluated this property in essential oil isolated by hydrodistillation from conventionally propagated $(\mathrm{CP})$ and in vitro propagated (IVP) K. galanga L rhizomes [34]. The antioxidant activity $\mathrm{IC}_{50}$ values assessed by DPPH radical scavenging were 26.0 and $19.5 \mu \mathrm{g} / \mathrm{mL}$ for CP and IVP oils, respectively. These results the oils' antioxidant activities are very high compared to this study.

\section{- CONCLUSION}

Generally, the high rhizome essential oil yield from selected K. galanga varieties was obtained by the maceration method. The tested oils comprise mainly compounds derived from shikimic acid, phenylpropanoid and exhibited high antioxidant 
activity with $\mathrm{IC}_{50}$ values, in the range of 85.24 to 89.19 $\mu \mathrm{g} / \mathrm{mL}$. Furthermore, the oils' major chemical component was ethyl trans-p-methoxycinnamate, and the most prevalent chemical constituents varied for varieties and isolation methods employed. Therefore, additional bioassays would be necessary to be done soon to uncover the biomedical of the oil. Furthermore, the complexation of the oil with cyclodextrin is expected to boost the complex's physicochemical properties and eventually increase the versatility of its applications.

\section{- AUTHOR CONTRIBUTIONS}

IWM conceived, designed, performed the experiments, and drafted the manuscript; IWM performed the GCMS analysis and corrected manuscript draft; NWM performed the antioxidant activities.

\section{- REFERENCES}

[1] Kumar, A., 2020, Phytochemistry, pharmacological activities and uses of traditional medicinal plant Kaempferia galanga L. - an Overview, J. Ethnopharmacol., 253, 112667.

[2] Preetha, T.S., Hemanthakumar, A.S., and Krishnan, P.N., 2016, A comprehensive review of Kaempferia Galanga L. (Zingiberaceae): A high sought medicinal plant in Tropical Asia, J. Med. Plants Stud., 4 (3), 270-276.

[3] Maurya, S.K., and Seth, A., 2014, Potential medicinal plants and traditional Ayurvedic approach towards Urticaria, an allergic skin disorder, Int. J. Pharm. Pharm. Sci., 6 (5), 172-177.

[4] Chan, E.W.C., Lim, Y.Y., Wong, L.F., Lianto, F.S., Wong, S.K., Lim, K.K., Joe, C.E., and Lim, T.Y., 2008, Antioxidant and tyrosinase inhibition properties of leaves and rhizomes of ginger species, Food Chem., 109 (3), 477-483.

[5] Elfahmi, Woerdenbag, H.J., and Kayser, O., 2014, Jamu: Indonesian traditional herbal medicine towards rational phytopharmacological use, J. Herb. Med., 4 (2), 51-73.

[6] Srivastava, N., Ranjana, Singh, S., Gupta, A.C., Shanker, K., Bawankule, D.U., and Luqman, S., 2019, Aromatic ginger (Kaempferia galanga L.) extracts with ameliorative and protective potential as a functional food, beyond its flavor and nutritional benefits, Toxicol. Rep., 6, 521-528.

[7] Ali, H., Yesmin, R., Satter, M.A., Habib, R., and Yeasmin, T., 2018, Antioxidant and antineoplastic activities of methanolic extract of Kaempferia galanga Linn. rhizome against Ehrlich ascites carcinoma cells, J. King Saud Univ., Sci., 30 (3), 386392.

[8] Amuamuta, A., Plengsuriyakarn, T., and NaBangchang, K., 2017, Anticholangiocarcinoma activity and toxicity of the Kaempferia galanga Linn. rhizome ethanolic extract, $B M C$ Complementary Altern. Med., 17 (1), 213.

[9] Plengsuriyakarn, T., Viyanant, V., Eursitthichai, V., Picha, P., Kupradinun, P., Itharat, A., and NaBangchang, K., 2012, Anticancer activities against cholangiocarcinoma, toxicity and pharmacological activities of Thai medicinal plants in animal models, BMC Complementary Altern. Med., 12, 23.

[10] Othman, R., Ibrahim, H., Mohd, M.A., Mustafa, M.R., and Awang, K., 2006, Bioassay-guided isolation of a vasorelaxant active compound from Kaempferia galanga L., Phytomedicine, 13 (1-2), 6166.

[11] Jagadish, P.C., Latha, K.P., Mudgal, J., and Nampurath, G.K., 2016, Extraction, characterization and evaluation of Kaempferia galanga L. (Zingiberaceae) rhizome extracts against acute and chronic inflammation in rats, $J$. Ethnopharmacol., 194, 434-439.

[12] Rostiana, O., Abdulah, A., Martono, B., Haryudin, W., and Aisyah, S., 1993, Pengaruh rimpang utama dan rimpang cabang terhadap pertumbuhan dan produksi tiga tipe kencur di kp. Citayam, Bul. Littro, 8 (2), 89-93.

[13] Rostiana, O.S.M., Rosita, H., Wawan, Supriadi, and Aisyah, S., 2003, Status pemuliaan tanaman kencur, Perkembangan Teknologi Tanaman Rempah dan Obat, 15 (2), 25-38.

[14] Barkatullah, Ibrar, M., Rauf, A., and Ur-Rahman, I., 2012, Physicochemical characterization of essential and fixed oils of Skimmia laureola and 
Zanthoxylum armatum, Middle-East J. Med. Plants Res., 1 (3), 51-58.

[15] Kumar, A., 2014, Physico-chemical and natural products investigations of essential oil from the rhizomes of Kaempferia galanga L., Chem. Sin., 5 (2), 91-94.

[16] Fan, S., Chang, J., Zong, Y., Hu, G., and Jia, J., 2018, GC-MS analysis of the composition of the essential oil from Dendranthema indicum Var. Aromaticum using three extraction methods and two columns, Molecules, 23 (3), 576.

[17] Shekhar, T.C., and Anju, G., 2014, Antioxidant activity by DPPH radical scavenging method of Ageratum conyzoides Linn. leaves, Am. J. Ethnomed., 1 (4), 244-249.

[18] Ali, H., Yesmin, R., Satter, M.A., Habib, R., and Yeasmin, T., 2018, Antioxidant and antineoplastic activities of methanolic extract of Kaempferia galanga Linn. rhizome against Ehrlich ascites carcinoma cells, J. King Saud Univ., Sci., 30 (3), 386-392.

[19] Huda, M.I., Daryon, E.D., and Muyassaroh, 2013, Minyak kencur dari rimpang kencur dengan variabel jumlah pelarut dan waktu maserasi, Jurnal Teknik Kimia, 8 (1), 1-6.

[20] Wong, K.C., Ong, K.S., and Lim, C.L., 1992, Composition of the essential oil of rhizomes of Kaempferia galanga L., Flavour Fragrance J., 7 (5), 263-266.

[21] Reveglia, P., Cimmino, A., Masi, M., Nocera, P., Berova, N., Ellestad, G., and Evidente, A., 2018, Pimarane diterpenes: Natural source, stereochemical configuration, and biological activity, Chirality, 30 (10), 1115-1134.

[22] Swapana, N., Tominaga, T., Elshamy, A.I., Ibrahim, M.A., Hegazy, M.E.F., Singh, C.B., Suenaga, M., Imagawa, H., Noji, M., and Umeyama, A., 2018, Kaemgalangol A: Unusual seco-isopimarane diterpenoid from aromatic ginger Kaempferia galanga, Fitoterapia, 129, 47-53.

[23] Jordá, T., and Puig, S., 2020, Regulation of ergosterol biosynthesis in Saccharomyces cerevisiae, Genes, 11 (7), 795.
[24] Hirsch, A.L., 2011, "Industrial Aspects of Vitamin

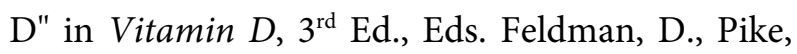
J.W., and Adams, J.S., Academic Press, San Diego, 73-93.

[25] Adianingsih, O.R., Widaryanto, E., Saitama, A., and Zaini, A.H., 2021, Analysis of bioactive compounds present in Kaempferia galanga rhizome collected from different regions of East Java, Indonesia, IOP Conf. Ser.: Earth Environ. Sci., 913, 012074.

[26] Yang, Y., Tian, S., Wang, F., and Li, Z., 2018, Chemical composition and antibacterial activity of Kaempferia galanga essential oil, Int. J. Agric. Biol., 20 (2), 457-462.

[27] Li, Y.C., Ji, H., Li, X.H., Zhang, H.X., and Li, H.T., 2017, Isolation of nematicidal constituents from essential oil of Kaempferia galanga L rhizome and their activity against Heterodera avenae Wollenweber, Trop. J. Pharm. Res., 16 (1), 59-65.

[28] Raina, A.P., Abraham, Z., and Sivaraj, N., 2015, Diversity analysis of Kaempferia galanga L. germplasm from South India using DIVA-GIS approach, Ind. Crops Prod., 69, 433-439.

[29] Srivastava, N., Ranjana, Singh, S., Gupta, A.C., Shanker, K., Bawankule, D.U., and Luqman, S., 2019, Aromatic ginger (Kaempferia galanga L.) extracts with ameliorative and protective potential as a functional food, beyond its flavor and nutritional benefits, Toxicol. Rep., 6, 521-528.

[30] Baharudin, M.K.A., Hamid, S.A., and Susanti, D., 2015, Chemical composition and antibacterial activity of essential oils from three aromatic plants of the Zingiberaceae family in Malaysia, J. Phys. Sci., 26 (1), 71-81.

[31] Tewtrakul, S., Yuenyongsawad, S., Kummee, S., and Atsawajaruwan, L., 2005, Chemical components and biological activities of volatile oil of Kaempferia galanga Linn, Songklanakarin J. Sci. Technol., 27 (Suppl. 2), 503-507.

[32] Wang, S.Y., Zhao, H., Xu, H.T., Han, X.D., Wu, Y.S., Xu, F.F., Yang, X.B., Göransson, U., and Liu, B., 2021, Kaempferia galanga L.: Progresses in phytochemistry, pharmacology, toxicology and 
ethnomedicinal uses, Front. Pharmacol., 12, 675350.

[33] Bhuiyan, M.N.I., Begum, J., and Anwar, M., 2013, Essential oils of leaves and rhizomes of Kaempferia galanga Linn, Chittagong Univ. J. Biol. Sci., 3 (1-2), 65-76.
[34] Sahoo, S., Parida, R., Singh, S., Padhy, R.N., and Nayak, S., 2014, Evaluation of yield, quality and antioxidant activity of essential oil of in vitro propagated Kaempferia galanga Linn, J. Acute Dis., $3(2), 124-130$. 